\title{
Kraśkiewicz-Pragacz modules and Pieri and dual Pieri rules for Schubert polynomials
}

\author{
Masaki Watanabe1fi \\ ${ }^{1}$ Graduate School of Mathematics, the University of Tokyo
}

\begin{abstract}
In their 1987 paper Kraśkiewicz and Pragacz defined certain modules, which we call KP modules, over the upper triangular Lie algebra whose characters are Schubert polynomials. In a previous work the author showed that the tensor product of Kraśkiewicz-Pragacz modules always has KP filtration, i.e. a filtration whose each successive quotients are isomorphic to KP modules. In this paper we explicitly construct such filtrations for certain special cases of these tensor product modules, namely $\mathcal{S}_{w} \otimes S^{d}\left(K^{i}\right)$ and $\mathcal{S}_{w} \otimes \bigwedge^{d}\left(K^{i}\right)$, corresponding to Pieri and dual Pieri rules for Schubert polynomials.

Résumé. Dans leur étude en 1987 Kraśkiewicz et Pragacz ont defini certains modules, que nous appelons modules KP, sur les algébres de Lie des matrices triangulaires supérieures, dont les caractéres sont les polynômes de Schubert. Dans une étude récente l'auteur a prouvé que les produits tensoriels de deux modules KP ont des filtrations KP, c'est-ádire des filtrations dont les quotients successifs sont des modules KP. Dans cet article nous construisons explicitement telles filtrations pour certains cas de ces produits tensoriels, á savoir $\mathcal{S}_{w} \otimes S^{d}\left(K^{i}\right)$ et $\mathcal{S}_{w} \otimes \bigwedge^{d}\left(K^{i}\right)$, correspondant aux formules de Pieri et de Pieri double pour les polynômes de Schubert.
\end{abstract}

Keywords. Schubert polynomials, Schubert functors, Kraśkiewicz-Pragacz modules, Schubert calculus

\section{Introduction}

Schubert polynomials are one of the main subjects in algebraic combinatorics. One of the tools for studying Schubert polynomials is the modules introduced by Kraśkiewicz and Pragacz. These modules, which here we call KP modules, are modules over the upper triangular Lie algebra and have the property that their characters with respect to the diagonal matrices are Schubert polynomials.

It is known that a product of Schubert polynomials is always a positive sum of Schubert polynomials. The previously known proof of this positivity property uses the geometry of the flag variety. In [Wat15a] the author showed that the tensor product of two KP modules always has a filtration by KP modules and thus gave a representation theoretic proof for this positivity. Although the proof there does not give explicit construction for the KP filtrations, it may provide a new viewpoint for the notorious SchubertLR problem, i.e. finding a combinatorial positive rule for the coefficient in the expansion of products of Schubert polynomials into a sum of Schubert polynomials.

\footnotetext{
†Email: mwata@ms.u-tokyo.ac.jp.

This work was supported by Grant-in-Aid for JSPS Fellows No. 15J05373.

1365-8050 (C) 2016 Discrete Mathematics and Theoretical Computer Science (DMTCS), Nancy, France
} 
There are some cases where the expansions of products of Schubert polynomials are explicitly known. Examples of such cases are the Pieri and the dual Pieri rules for Schubert polynomials conjectured in [BB93] and proved in [Win98] (also appearing with different formulations in [Las82] and [Sot96]). They are the cases where one of the Schubert polynomials is a complete symmetric function $h_{d}\left(x_{1}, \ldots, x_{i}\right)$ or an elementary symmetric function $e_{d}\left(x_{1}, \ldots, x_{i}\right)$. The purpose of this paper is to investigate the structure of tensor product modules corresponding to these products and to give an explicit construction of KP filtrations for these modules.

The structure of this paper is as follows. In Section 2 we prepare some definitions and results on Schubert polynomials and KP modules. In Section 3 we review the Pieri and dual Pieri rules for Schubert polynomials. In Section 4 we give an explicit construction for KP filrartions of the corresponding tensor product modules $\mathcal{S}_{w} \otimes S^{d}\left(K^{i}\right)$ and $\mathcal{S}_{w} \otimes \bigwedge^{d}\left(K^{i}\right)$. In Section 5 we sketch the proof of the main result.

\section{Preliminary}

Let $\mathbb{N}$ be the set of all positive integers. By a permutation $w$ we mean a bijection from $\mathbb{N}$ to itself which fixes all but finitely many points. The graph of a permutation $w$ is the $\operatorname{set}\{(i, w(i)): i \in \mathbb{N}\} \subset \mathbb{N}^{2}$. For $i<j$, let $t_{i j}$ denote the permutation which exchanges $i$ and $j$ and fixes all other points. Let $s_{i}=t_{i, i+1}$. For a permutation $w$, let $\ell(w)=\#\{i<j: w(i)>w(j)\}$. For a permutation $w$ and $p<q$, if $\ell\left(w t_{p q}\right)=\ell(w)+1$ we write $w t_{p q}>w$. It is well known that this condition is equivalent to saying that $w(p)<w(q)$ and there exists no $p<r<q$ satisfying $w(p)<w(r)<w(q)$. For a permutation $w$ let $I(w)=\{(i, j): i<j, w(i)>w(j)\}$.

For a polynomial $f=f\left(x_{1}, x_{2}, \ldots\right)$ and $i \in \mathbb{N}$ define $\partial_{i} f=\frac{f-s_{i} f}{x_{i}-x_{i+1}}$. For a permutation $w$ we can assign its Schubert polynomial $\mathfrak{S}_{w} \in \mathbb{Z}\left[x_{1}, x_{2}, \ldots\right]$ which is recursively defined by

- $\mathfrak{S}_{w}=x_{1}^{n-1} x_{2}^{n-2} \cdots x_{n-1}$ if $w(1)=n, w(2)=n-1, \ldots, w(n)=1$ and $w(i)=i(i>n)$, and

- $\mathfrak{S}_{w s_{i}}=\partial_{i} \mathfrak{S}_{w}$ if $\ell\left(w s_{i}\right)<\ell(w)$.

Hereafter let us fix a positive integer $n$. Let $S^{(n)}=\{w$ : permutation, $w(n+1)<w(n+2)<\cdots\}$. Note that if $w \in S^{(n)}$ then $I(w) \subset\{1, \ldots, n\} \times \mathbb{N}$. Let $K$ be a field of characteristic zero. Let $\mathfrak{b}=\mathfrak{b}_{n}$ denote the Lie algebra of all $n \times n$ upper triangular matrices over $K$. For a $\mathcal{U}(\mathfrak{b})$-module $M$ and $\lambda=\left(\lambda_{1}, \ldots, \lambda_{n}\right) \in \mathbb{Z}^{n}$, let $M_{\lambda}=\left\{m \in M: h m=\langle\lambda, h\rangle m\left(\forall h=\operatorname{diag}\left(h_{1}, \ldots, h_{n}\right)\right)\right\}$ where $\langle\lambda, h\rangle=\sum_{i} \lambda_{i} h_{i}$. If $M$ is a direct sum of these $M_{\lambda}$ then we say that $M$ is a weight module and we define $\operatorname{ch}(M)=\sum_{\lambda} \operatorname{dim} M_{\lambda} x^{\lambda}$ where $x^{\lambda}=x_{1}^{\lambda_{1}} \cdots x_{n}^{\lambda_{n}}$. For $1 \leq i \leq j \leq n$ let $e_{i j} \in \mathfrak{b}$ be the matrix with 1 at the $(i, j)$-th position and all other coordinates 0 .

Let $V$ be a vector space spanned by a basis $\left\{u_{i j}: 1 \leq i \leq n, j \in \mathbb{N}\right\}$. Let $T=\bigwedge^{\bullet} V$. The Lie algebra $\mathfrak{b}$ acts on $V$ by $e_{p q} u_{i j}=\delta_{i q} u_{p j}$ and thus on $T$. For $w \in S^{(n)}$ let $u_{w}=\bigwedge_{(i, j) \in I(w)} u_{i j} \in T$. The Kraśkiewicz-Pragacz module $\mathcal{S}_{w}$ (or the KP module for short) associated with $w$ is the $\mathfrak{b}$-submodule of $T$ generated by $u_{w}$. In [KP04] Kraśkiewicz and Pragacz showed the following:

Theorem 2.1 ([KP04, Remark 1.6 and Theorem 4.1]) $\mathcal{S}_{w}$ is a weight module and $\operatorname{ch}\left(\mathcal{S}_{w}\right)=\mathfrak{S}_{w}$.

Example 2.2 If $w=s_{i}$, then $u_{w}=u_{i, i+1}$ and thus $\mathcal{S}_{w}=\bigoplus_{1 \leq j \leq i} K u_{j, i+1} \cong \bigoplus_{1 \leq j \leq i} K u_{j}=: K^{i}$ where $\mathfrak{b}$ acts by $e_{p q} u_{j}=\delta_{q j} u_{p}$.

A $K P$ filtration of a $\mathfrak{b}$-module $M$ is a filtration $0=M_{0} \subset \cdots \subset M_{r}=M$ such that each $M_{i} / M_{i-1}$ is isomorphic to some KP module. 


\section{Pieri and dual Pieri rules for Schubert polynomials}

Definition 3.1 For $w \in S_{\infty}, i \geq 1$ and $d \geq 0$, let

$$
X_{i, d}(w)=\left\{t_{p_{1} q_{1}} t_{p_{2} q_{2}} \cdots t_{p_{d} q_{d}}: p_{j} \leq i, q_{j}>i, w_{1} \lessdot w_{2} \lessdot \cdots, w_{1}\left(p_{1}\right)<w_{2}\left(p_{2}\right)<\cdots\right\}
$$

and

$$
Y_{i, d}(w)=\left\{t_{p_{1} q_{1}} t_{p_{2} q_{2}} \cdots t_{p_{d} q_{d}}: p_{j} \leq i, q_{j}>i, w_{1} \lessdot w_{2} \lessdot \cdots, w_{1}\left(p_{1}\right)>w_{2}\left(p_{2}\right)>\cdots\right\}
$$

where $w_{1}=w, w_{2}=w t_{p_{1} q_{1}}, w_{3}=w t_{p_{1} q_{1}} t_{p_{2} q_{2}} \cdots$.

Note that the condition above for $X_{i, d}(w)$ (resp. $Y_{i, d}(w)$ ) also implies $w_{1}\left(q_{1}\right)<w_{2}\left(q_{2}\right)<\cdots$ (resp. $\left.w_{1}\left(q_{1}\right)>w_{2}\left(q_{2}\right)>\cdots\right)$. Also note that the condition for $X_{i, d}(w)\left(\right.$ resp. $\left.Y_{i, d}(w)\right)$ implies that $q_{1}, \ldots, q_{d}$ (resp. $\left.p_{1}, \ldots, p_{d}\right)$ are all different.

Theorem 3.2 (conjectured in [BB93] and proved in [Win98] ) [i) We have

$$
\mathfrak{S}_{w} \cdot h_{d}\left(x_{1}, \ldots, x_{i}\right)=\sum_{\zeta \in X_{i, d}(w)} \mathfrak{S}_{w \zeta}
$$

and

$$
\mathfrak{S}_{w} \cdot e_{d}\left(x_{1}, \ldots, x_{i}\right)=\sum_{\zeta \in Y_{i, d}(w)} \mathfrak{S}_{w \zeta} .
$$

where $h_{d}$ and $e_{d}$ denote the complete and elementary symmetric functions respectively.

Note here that the sums above are multiplicity-free: that is, the permutation $\zeta$ actually determines uniquely its decomposition into transpositions satisfying the conditions in Definition 3.1. So we can write without ambiguity, for example "for $\zeta=t_{p_{1} q_{1}} \cdots t_{p_{d} q_{d}} \in X_{i, d}(w)$ define (something) as (some formula involving $p_{j}$ and $q_{j}$ )". Hereafter if we write such we will always assume the conditions in Definition 3.1

\section{Explicit Pieri and dual Pieri rules for KP modules}

The author showed in [Wat15a] that the tensor product of KP modules always has a KP filtration. Since $S^{d}\left(K^{i}\right)$ and $\bigwedge^{d}\left(K^{i}\right)$ are special cases of KP modules for any $1 \leq i \leq n$ and $d \geq 1, \mathcal{S}_{w} \otimes S^{d}\left(K^{i}\right)$ and $\mathcal{S}_{w} \otimes \bigwedge^{d}\left(K^{i}\right)\left(w \in S^{(n)}\right)$ have KP filtrations. In this section we give an explicit construction of these filtrations.

For integers $p \leq q$ we define an operator $e_{q p}^{\prime}$ acting on $T$ as $e_{q p}^{\prime}\left(u_{a_{1} b_{1}} \wedge u_{a_{2} b_{2}} \wedge \cdots\right)=\sum_{k}(\cdots \wedge$ $\left.\delta_{p b_{k}} u_{a_{k} q} \wedge \cdots\right)$. Let these operators act on $T \otimes S^{d}\left(K^{i}\right)$ and $T \otimes \bigwedge^{d}\left(K^{i}\right)$ by applying them on the left-hand side tensor component. We also define operators $\mu_{j}$ on $T \otimes \bigotimes^{a}\left(K^{i}\right) \rightarrow T \otimes \bigotimes^{a-1}\left(K^{i}\right)$ by $u \otimes\left(v_{1} \otimes v_{2} \otimes \cdots\right) \mapsto\left(\iota_{j}\left(v_{1}\right) \wedge u\right) \otimes\left(v_{2} \otimes v_{3} \otimes \cdots\right)$ where $\iota_{j}: u_{p} \mapsto u_{p j}$. We denote the restrictions of $\mu_{j}$ to $T \otimes S^{a}\left(K^{i}\right)$ and $T \otimes \bigwedge^{a}\left(K^{i}\right)$ by the same symbol. Note that $e_{r s}^{\prime}$ and $\mu_{j}$ give $\mathfrak{b}$-endomorphisms on $T \otimes S^{\bullet}\left(K^{i}\right)$ and $T \otimes \bigwedge^{\bullet}\left(K^{i}\right)$.

(i) Also appears with different formulations in [Las82] and [Sot96]. 
For a permutation $z$ and $p<q$ let $m_{p q}(z)=\#\{r>q: z(p)<z(r)<z(q)\}$ and $m_{q p}^{\prime}(z)=\#\{r<$ $p: z(p)<z(r)<z(q)\}$. For $\zeta=t_{p_{1} q_{1}} \cdots t_{p_{d} q_{d}} \in X_{i, d}(w)$ (resp. $\left.Y_{i, d}(w)\right)$ define

$$
\begin{aligned}
v_{\zeta} & =\left(\prod_{j} e_{p_{j} q_{j}}^{m_{p_{j} q_{j}}\left(w_{j}\right)} u_{w}\right) \otimes \prod_{j} u_{p_{j}} \\
& =\left(\prod_{j} e_{p_{j} q_{j}}^{m_{p_{j} q_{j}}\left(w_{j}\right)} u_{w}\right) \otimes\left(\sum_{\sigma \in S_{d}} u_{p_{\sigma}(1)} \otimes \cdots \otimes u_{p_{\sigma}(d)}\right) \in \mathcal{S}_{w} \otimes S^{d}\left(K^{i}\right)
\end{aligned}
$$

(resp.

$$
\begin{aligned}
v_{\zeta} & =\left(\prod_{j} e_{p_{j} q_{j}}^{m_{p_{j} q_{j}}\left(w_{j}\right)} u_{w}\right) \otimes \bigwedge_{j} u_{p_{j}} \\
& =\left(\prod_{j} e_{p_{j} q_{j}}^{m_{p_{j} q_{j}}\left(w_{j}\right)} u_{w}\right) \otimes\left(\sum_{\sigma \in S_{d}} \operatorname{sgn} \sigma \cdot u_{p_{\sigma}(1)} \otimes \cdots \otimes u_{p_{\sigma}(d)}\right) \in \mathcal{S}_{w} \otimes \bigwedge^{d}\left(K^{i}\right)
\end{aligned}
$$

) where $w_{j}=w t_{p_{1} q_{1}} \cdots t_{p_{j-1} q_{j-1}}$ as in Definition 3.1 (note that these are also well-defined if some $q_{j}$ are greater than $n$, since in such case $m_{p_{j} q_{j}}\left(w_{j}\right)=0$ ). Also, for such $\zeta$, define a $\mathfrak{b}$-homomorphism $T \otimes \otimes^{d}\left(K^{i}\right) \rightarrow T$ by $\phi_{\zeta}=\mu_{q_{d}} \cdots \mu_{q_{1}} \cdot \prod_{j}\left(e_{q_{j} p_{j}}^{\prime}\right)^{m_{q_{j} p_{j}}^{\prime}\left(w_{j}\right)}$.

Proposition 4.1 For $\zeta, \zeta^{\prime} \in X_{i, d}(w)$ (resp. $\left.Y_{i, d}(w)\right)$,

- $\phi_{\zeta}\left(v_{\zeta}\right)$ is a nonzero multiple of $u_{w \zeta} \in T$, and

- $\phi_{\zeta^{\prime}}\left(v_{\zeta}\right)=0$ if $(w \zeta)^{-1} \underset{\text { lex }}{<}\left(w \zeta^{\prime}\right)^{-1}\left(\right.$ resp. $\left.(w \zeta)^{-1} \underset{\operatorname{rlex}}{<}\left(w \zeta^{\prime}\right)^{-1}\right)$.

A sketched proof for this proposition is given in the next section.

For a $\mathfrak{b}$-module $M$ and elements $x, y, \ldots, z \in M$ let $\langle x, y, \ldots, z\rangle$ denote the submodule generated by these elements. Consider the sequence of submodules

$$
0 \subset\left\langle v_{\zeta_{1}}\right\rangle \subset\left\langle v_{\zeta_{1}}, v_{\zeta_{2}}\right\rangle \subset \cdots \subset\left\langle v_{\zeta}: \zeta \in X_{i, d}(w)\left(\operatorname{resp} . Y_{i, d}(w)\right)\right\rangle
$$

inside $\mathcal{S}_{w} \otimes S^{d}\left(K^{i}\right)\left(\right.$ resp. $\left.\mathcal{S}_{w} \otimes \bigwedge^{d}\left(K^{i}\right)\right)$, where $\zeta_{1}, \zeta_{2}, \ldots \in X_{i, d}(w)$ (resp. $Y_{i, d}(w)$ ) are all the elements ordered so that $\left(w \zeta_{1}\right)^{-1} \underset{\text { lex }}{<}\left(w \zeta_{2}\right)^{-1} \underset{\text { lex }}{<} \cdots$ (resp. $\left.\left(w \zeta_{1}\right)^{-1} \underset{\text { rlex }}{<}\left(w \zeta_{2}\right)^{-1} \underset{\text { rlex }}{<} \cdots\right)$. From the proposition we see that there is a surjection $\left\langle v_{\zeta_{1}}, \cdots, v_{\zeta_{j}}\right\rangle /\left\langle v_{\zeta_{1}}, \cdots, v_{\zeta_{j-1}}\right\rangle \rightarrow \mathcal{S}_{w \zeta_{j}}$ induced from $\phi_{\zeta_{j}}$. Thus we have

$$
\operatorname{dim}\left(\mathcal{S}_{w} \otimes S^{d}\left(K^{i}\right)\right) \geq \operatorname{dim}\left\langle v_{\zeta}: \zeta \in X_{i, d}(w)\right\rangle \geq \sum_{\zeta \in X_{i, d}(w)} \operatorname{dim} \mathcal{S}_{w \zeta}=\operatorname{dim}\left(\mathcal{S}_{w} \otimes S^{d}\left(K^{i}\right)\right)
$$

and

$$
\operatorname{dim}\left(\mathcal{S}_{w} \otimes \bigwedge^{d}\left(K^{i}\right)\right) \geq \operatorname{dim}\left\langle v_{\zeta}: \zeta \in Y_{i, d}(w)\right\rangle \geq \sum_{\zeta \in Y_{i, d}(w)} \operatorname{dim} \mathcal{S}_{w \zeta}=\operatorname{dim}\left(\mathcal{S}_{w} \otimes \bigwedge^{d}\left(K^{i}\right)\right)
$$

respectively, where the last equalities are by Theorem 3.2 . So the equality must hold everywhere, and thus the surjection above is in fact an isomorphism. So we get: 
Theorem 4.2 Let $M=\mathcal{S}_{w} \otimes S^{d}\left(K^{i}\right)$ (resp. $\mathcal{S}_{w} \otimes \bigwedge^{d}\left(K^{i}\right)$ ). Define $v_{\zeta}$ and $\phi_{\zeta}$ as above. Then $M$ is generated by $\left\{v_{\zeta}: \zeta \in X_{i, d}(w)\right.$ (resp. $\left.\left.Y_{i, d}(w)\right)\right\}$ as a $\mathfrak{b}$-module and

$$
0 \subset\left\langle v_{\zeta_{1}}\right\rangle \subset\left\langle v_{\zeta_{1}}, v_{\zeta_{2}}\right\rangle \subset \cdots \subset\left\langle v_{\zeta}: \zeta \in X_{i, d}(w)\left(\text { resp. } Y_{i, d}(w)\right)\right\rangle
$$

gives a KP filtration of $M$, where $\zeta_{1}, \zeta_{2}, \ldots \in X_{i, d}(w)$ (resp. $Y_{i, d}(w)$ ) are all the elements ordered increasingly by the lexicographic (resp. reverse lexicographic) ordering on $(w \zeta)^{-1}$. The explicit isomorphism $\left\langle v_{\zeta_{1}}, \cdots, v_{\zeta_{j}}\right\rangle /\left\langle v_{\zeta_{1}}, \cdots, v_{\zeta_{j-1}}\right\rangle \cong \mathcal{S}_{w \zeta_{j}}$ is given by $\phi_{\zeta_{j}}$ defined above.

Remark 4.3 In [Wat15b] we related KP modules with the notion of highest weight categories ([CPS88]) as follows. For $\Lambda^{\prime} \subset \mathbb{Z}^{n}$ let $\mathcal{C}_{\Lambda^{\prime}}$ be the category of weight $\mathfrak{b}_{n}$-modules whose weights are all in $\Lambda^{\prime}$. Then if $\Lambda^{\prime}$ is an order ideal with respect to a certain ordering on $\mathbb{Z}^{n}$ then $\mathcal{C}_{\Lambda^{\prime}}$ has a structure of highest weight category whose standard objects are KP modules. One of the axioms required for a highest weight category states that the projective objects should have filtrations by standard objects.

It can be shown that the projective cover of the one dimensional $\mathfrak{b}_{n}$-module $K_{\lambda}$ with weight $\lambda=$ $\left(\lambda_{1}, \ldots, \lambda_{n}\right) \in \mathbb{Z}_{\geq 0}^{n}$ in the category $\mathcal{C}_{\mathbb{Z}_{\geq 0}^{n}}$ is given by $S^{\lambda_{1}}\left(K^{1}\right) \otimes \cdots \otimes S^{\lambda_{n}}\left(K^{n}\right)$. Thus Theorem 4.2 gives a proof to the fact that the indecomposable projective modules in $\mathcal{C}_{\mathbb{Z}_{\geq 0}^{n}}$ have KP filtrations, which leads to a different proof from the one in [Wat15b §3] for the axiom mentioned above (we do not need these results about highest weight structure for $\mathfrak{b}$-modules in the proof of Theorem 4.2).

\section{Skeched Proof of Proposition 4.1}

Lemma 5.1 ( Implicit in [Wat15b, Proof of Theorem 5.1] ) (ii) Let $w \in S^{(n)}$ and $i \geq 1$. For p, $p^{\prime} \leq$ $i$ and $q, q^{\prime}>i$ such that $\ell\left(w t_{p q}\right)=\ell\left(w t_{p^{\prime} q^{\prime}}\right)=\ell(w)+1$ (i.e. $\left.t_{p q}, t_{p^{\prime} q^{\prime}} \in X_{i, 1}(w)\right)$, if $u_{p q^{\prime}} \wedge$ $e_{p q}^{m_{p q}(w)}\left(e_{q^{\prime} p^{\prime}}^{\prime}\right)^{m_{q^{\prime} p^{\prime}}^{\prime}(w)} u_{w} \neq 0$ then $w\left(p^{\prime}\right) \geq w(p)$ and $w\left(q^{\prime}\right) \geq w(q)$, and if $(p, q)=\left(p^{\prime}, q^{\prime}\right)$ it is a nonzero multiple of $u_{w t_{p q}}$.

Lemma 5.2 Let $w$ be a permutation, $i \geq 1$ and $d \geq 0$. Let $\zeta=t_{p_{1} q_{1}} \cdots t_{p_{d} q_{d}} \in X_{i, d}(w)$ (resp. $\left.Y_{i, d}(w)\right)$ and $1 \leq a \leq d$. Suppose that there exists no $b<a$ satisfying $p_{b}=p_{a}\left(\right.$ resp. $\left.q_{b}=q_{a}\right)$. Then $m_{p_{a} q_{a}}\left(w_{a}\right)=m_{p_{a} q_{a}}(w)$ and $m_{q_{a} p_{a}}^{\prime}\left(w_{a}\right)=m_{q_{a} p_{a}}^{\prime}(w)$ where $w_{a}=w t_{p_{1} q_{1}} \cdots t_{p_{a-1} q_{a-1}}$ as in Definition 3.1

Proof of Proposition 4.1; The proofs for the cases of $X_{i, d}(w)$ and $Y_{i, d}(w)$ are similar. Here we give a proof for the case of $X_{i, d}(w)$.

We assume $(w \zeta)^{-1} \underset{\text { lex }}{\leq}\left(w \zeta^{\prime}\right)^{-1}$ (resp. $(w \zeta)^{-1} \underset{\text { rlex }}{\leq}\left(w \zeta^{\prime}\right)^{-1}$ ) and show that $\phi_{\zeta^{\prime}}\left(v_{\zeta}\right)=0$ unless $\zeta^{\prime}=\zeta$ and $\phi_{\zeta}\left(v_{\zeta}\right)$ is a nonzero multiple of $u_{w \zeta}$. Let $\zeta=t_{p_{1} q_{1}} \cdots t_{p_{d} q_{d}}$ and $\zeta^{\prime}=t_{p_{1}^{\prime} q_{1}^{\prime}} \cdots t_{p_{d}^{\prime} q_{d}^{\prime}}$ as in Definition 3.1. We write $w_{a}=w t_{p_{1} q_{1}} \cdots t_{p_{a-1} q_{a-1}}$ and $w_{a}^{\prime}=w t_{p_{1}^{\prime} q_{1}^{\prime}} \cdots t_{p_{a-1}^{\prime} q_{a-1}^{\prime}}$.

For $\zeta=\prod_{j} t_{p_{j} q_{j}}$ and $\zeta^{\prime}=\prod_{j} t_{p_{j}^{\prime} q_{j}^{\prime}}$ in $X_{i, d}(w)$ we have

$$
\phi_{\zeta^{\prime}}\left(v_{\zeta}\right)=\sum_{\sigma \in S_{d}}\left(u_{p_{\sigma(d)} q_{d}^{\prime}} \wedge \cdots \wedge u_{p_{\sigma(1)} q_{1}^{\prime}} \wedge\left(\prod_{j} E_{j} \prod_{j} E_{j}^{\prime} \cdot u_{w}\right)\right)
$$

where $E_{j}=e_{p_{j} q_{j}}^{m_{p_{j} q_{j}}\left(w_{j}\right)}$ and $E_{j}^{\prime}=\left(e_{q_{j}^{\prime} p_{j}^{\prime}}^{\prime}\right)^{m_{q_{j}^{\prime} p_{j}^{\prime}}^{\prime}\left(w_{j}^{\prime}\right)}$.

(ii) In a revised version (in preparation) of [Wat15b this will appear as a separate lemma. 
If $w\left(p_{1}\right)<w\left(p_{1}^{\prime}\right)$, then $(w \zeta)^{-1}\left(w\left(p_{1}\right)\right)=q_{1}>p_{1}=\left(w \zeta^{\prime}\right)^{-1}\left(w\left(p_{1}\right)\right)$ and $(w \zeta)^{-1}(j)=w^{-1}(j)=$ $\left(w \zeta^{\prime}\right)^{-1}(j)$ for all $j<w\left(p_{1}\right)$, and this contradicts the assumption $(w \zeta)^{-1} \underset{\operatorname{lex}}{\leq}\left(w \zeta^{\prime}\right)^{-1}$. Thus $w\left(p_{1}\right) \geq$ $w\left(p_{1}^{\prime}\right)$.

Fix $\sigma \in S_{d}$. Let $1 \leq a \leq d$ be minimal such that $p_{a}=p_{\sigma(1)}$. Then we have

$$
\begin{aligned}
& u_{p_{\sigma(d)} q_{d}^{\prime}} \wedge \cdots \wedge u_{p_{\sigma(1)} q_{1}^{\prime}} \wedge\left(\prod_{j} E_{j} \prod_{j} E_{j}^{\prime} \cdot u_{w}\right) \\
& =u_{p_{\sigma(d)} q_{d}^{\prime}} \wedge \cdots \wedge u_{p_{\sigma(2)} q_{2}^{\prime}} \wedge \prod_{j \neq a} E_{j} \prod_{j \neq 1} E_{j}^{\prime} \cdot\left(u_{p_{\sigma(1)} q_{1}^{\prime}} \wedge E_{a} E_{1}^{\prime} u_{w}\right) \\
& =u_{p_{\sigma(d)} q_{d}^{\prime}} \wedge \cdots \wedge u_{p_{\sigma(2)} q_{2}^{\prime}} \wedge \prod_{j \neq a} E_{j} \prod_{j \neq 1} E_{j}^{\prime} \cdot\left(u_{p_{a} q_{1}^{\prime}} \wedge e_{p_{a} q_{a}}^{m_{p_{a} q_{a}}\left(w_{a}\right)}\left(e_{q_{1}^{\prime} p_{1}^{\prime}}^{\prime}\right)^{m_{q_{1}^{\prime} p_{1}^{\prime}}^{\prime}\left(w_{1}^{\prime}\right)} u_{w}\right) \\
& =u_{p_{\sigma(d)} q_{d}^{\prime}} \wedge \cdots \wedge u_{p_{\sigma(2)} q_{2}^{\prime}} \wedge \prod_{j \neq a} E_{j} \prod_{j \neq 1} E_{j}^{\prime} \cdot\left(u_{p_{a} q_{1}^{\prime}} \wedge e_{p_{a} q_{a}}^{m_{p_{a} q_{a}}(w)}\left(e_{q_{1}^{\prime} p_{1}^{\prime}}^{\prime}\right)^{m_{q_{1}^{\prime} p_{1}^{\prime}}^{\prime}(w)} u_{w}\right)
\end{aligned}
$$

where the last equality is by Lemma 5.2 (note that $w_{1}^{\prime}=w$ by definition).

First consider the case $w\left(p_{1}\right)>w\left(p_{1}^{\prime}\right)$. We show that the summand in $(*)$ vanishes for all $\sigma$. It suffices to show $u_{p_{a} q_{1}^{\prime}} \wedge e_{p_{a} q_{a}}^{m_{p_{a} q_{a}}(w)}\left(e_{q_{1}^{\prime} p_{1}^{\prime}}^{\prime}\right)^{m_{q_{1}^{\prime} p_{1}^{\prime}}^{\prime}(w)} u_{w}=0$. We have $w\left(p_{a}\right)=w_{a}\left(p_{a}\right) \geq w\left(p_{1}\right)>w\left(p_{1}^{\prime}\right)$. Thus by Lemma 5.1 we see $u_{p_{a} q_{1}^{\prime}} \wedge e_{p_{a} q_{a}}^{m_{p_{a} q_{a}}(w)}\left(e_{q_{1}^{\prime} p_{1}^{\prime}}^{\prime}\right)^{m_{q_{1}^{\prime} p_{1}^{\prime}}^{\prime}(w)} u_{w}=0$.

Next consider the case $w\left(p_{1}\right)=w\left(p_{1}^{\prime}\right)$. Here $(w \zeta)^{-1}\left(w\left(p_{1}\right)\right)=q_{1},\left(w \zeta^{\prime}\right)^{-1}\left(w\left(p_{1}\right)\right)=q_{1}^{\prime}$ and $(w \zeta)^{-1}(j)=w^{-1}(j)=\left(w \zeta^{\prime}\right)^{-1}(j)$ for all $j<w\left(p_{1}\right)$, and thus $q_{1} \leq q_{1}^{\prime}$ by the hypothesis. First consider the case $q_{1}<q_{1}^{\prime}$. Then since $w t_{p_{1} q_{1}}, w t_{p_{1}^{\prime} q_{1}^{\prime}}>w$ it follows that $w\left(q_{1}^{\prime}\right)<w\left(q_{1}\right)$. So $w\left(q_{1}^{\prime}\right)<$ $w\left(q_{1}\right) \leq w\left(q_{a}\right)$ and again by Lemma 5.1 we see $u_{p_{a} q_{1}^{\prime}} \wedge e_{p_{a} q_{a}}^{m_{p_{a}}(w)}\left(e_{q_{1}^{\prime} p_{1}^{\prime}}^{\prime}\right)^{m_{q_{1}^{\prime} p_{1}^{\prime}}^{\prime}(w)} u_{w}=0$.

Now consider the case $w\left(p_{1}\right)=w\left(p_{1}^{\prime}\right)$ and $q_{1}=q_{1}^{\prime}$. Then if $a>1$ we see

$$
u_{p_{a} q_{1}^{\prime}} \wedge e_{p_{a} q_{a}}^{m_{p_{a} q_{a}}(w)}\left(e_{q_{1}^{\prime} p_{1}^{\prime}}^{\prime}\right)^{m_{q_{1}^{\prime} p_{1}^{\prime}}^{\prime}(w)} u_{w}=0
$$

by Lemma 5.1 since $w\left(q_{1}^{\prime}\right)=w\left(q_{1}\right)<w\left(q_{a}\right)$ in such case. So the only remaining summands in $(*)$ are the ones with $a=1$, i.e. $p_{\sigma(1)}=p_{1}$. It is easy to see that the sum of such summands is a nonzero multiple of the sum of terms with $\sigma(1)=1$. If $\sigma(1)=1$ we have, by the latter part of Lemma 5.1.

$$
\begin{aligned}
& u_{p_{\sigma(d)} q_{d}^{\prime}} \wedge \cdots \wedge u_{p_{\sigma(1)} q_{1}^{\prime}} \wedge\left(\prod_{j} E_{j} \prod_{j} E_{j}^{\prime} \cdot u_{w}\right) \\
& =u_{p_{\sigma(d)} q_{d}^{\prime}} \wedge \cdots \wedge u_{p_{\sigma(2)} q_{2}^{\prime}} \wedge \prod_{j \neq 1} e_{p_{j} q_{j}}^{m_{p_{j} q_{j}}\left(w_{j}\right)} \prod_{j \neq 1}\left(e_{q_{j}^{\prime} p_{j}^{\prime}}^{\prime}\right)^{m_{q_{j}^{\prime} p_{j}^{\prime}}^{\prime}\left(w_{j}^{\prime}\right)}\left(u_{p_{1} q_{1}} \wedge e_{p_{1} q_{1}}^{m_{p_{1} q_{1}}(w)}\left(e_{q_{1} p_{1}}^{\prime}\right)^{m_{q_{1} p_{1}}^{\prime}(w)} u_{w}\right) \\
& =(\neq 0 \text { const. }) \cdot u_{p_{\sigma(d)} q_{d}^{\prime}} \wedge \cdots \wedge u_{p_{\sigma(2)} q_{2}^{\prime}} \wedge \prod_{j \neq 1} e_{p_{j} q_{j}}^{m_{p_{j} q_{j}}\left(w_{j}\right)} \prod_{j \neq 1}\left(e_{q_{j}^{\prime} p_{j}^{\prime}}^{\prime}\right)^{m_{q_{j}^{\prime} p_{j}^{\prime}}^{\prime}\left(w_{j}^{\prime}\right)} u_{w t_{p_{1} q_{1}}} .
\end{aligned}
$$

So, working inductively on $d$ (using $w t_{p_{1} q_{1}}, t_{p_{2} q_{2}} \cdots t_{p_{d} q_{d}}$ and $t_{p_{2}^{\prime} q_{2}^{\prime}} \cdots t_{p_{d}^{\prime} q_{d}^{\prime}}$ in places for $w, \zeta$ and $\zeta^{\prime}$ respectively: note that if $\left(p_{1}, q_{1}\right)=\left(p_{1}^{\prime}, q_{1}^{\prime}\right)$ then $(w \zeta)^{-1} \leq\left(w \zeta^{\prime}\right)^{-1}$ implies $\left(\left(w t_{p_{1} q_{1}}\right) \cdot t_{p_{2} q_{2}} \cdots t_{p_{d} q_{d}}\right)^{-1}=$ $\left.(w \zeta)^{-1} \underset{\operatorname{lex}}{\leq}\left(w \zeta^{\prime}\right)^{-1}=\left(\left(w t_{p_{1} q_{1}}\right) \cdot t_{p_{2}^{\prime} q_{2}^{\prime}} \cdots t_{p_{d}^{\prime} q_{d}^{\prime}}\right)^{-1}\right)$ we see that 
- $\left(u_{p_{\sigma(d)} q_{d}^{\prime}} \wedge \cdots \wedge u_{p_{\sigma(1)} q_{1}^{\prime}}\right) \wedge \prod_{j} e_{p_{j} q_{j}}^{m_{p_{j}}\left(w_{j}\right)} \prod_{j}\left(e_{q_{j}^{\prime} p_{j}^{\prime}}^{\prime}\right)^{m_{q_{j}^{\prime} p_{j}^{\prime}}^{\prime}\left(w_{j}^{\prime}\right)} u_{w}$ vanishes if $(w \zeta)^{-1} \underset{\text { lex }}{<}\left(w \zeta^{\prime}\right)^{-1}$, or if $\zeta^{\prime}=\zeta$ and $\sigma \neq \mathrm{id}$, and

- if $\zeta^{\prime}=\zeta$ and $\sigma=$ id then it is a nonzero multiple of $u_{w \zeta}$.

This finishes the proof.

\section{References}

[BB93] N. Bergeron and S. Billey. RC-graphs and Schubert polynomials. Experiment. Math., 2:257-269, 1993.

[BB05] A. Björner and F. Brenti. Combinatorics of Coxeter Groups. Springer, 2005.

[CPS88] E. Cline, B. Parshall, and L. Scott. Finite-dimensional algebras and highest weight categories. J. Reine Angew. Math., 391:85-99, 1988.

[KP87] W. Kraśkiewicz and P. Pragacz. Foncteurs de Schubert. C. R. Acad. Sci. Paris Sér. I Math., 304(9):209-211, 1987.

[KP04] Witold Kraśkiewicz and Piotr Pragacz. Schubert functors and Schubert polynomials. Eur. J. Comb., 25(8):1327-1344, 2004.

[Las82] A. Lascoux. Polynômes de Schubert. C. R. Acad. Sci. Paris Sér. I Math., 294(13):447-450, 1982.

[Sot96] F. Sottile. Pieri's formula for flag manifolds and Schubert polynomials. Ann. Inst. Fourier, Grenoble, 46(1):89-110, 1996.

[Wat15a] M. Watanabe. Tensor product of Kraśkiewicz-Pragacz modules. J. Algebra, 443:422-429, 2015.

[Wat15b] M. Watanabe. Kraśkiewicz-Pragacz modules and Ringel duality. preprint, arXiv: $1504.04657 \mathrm{v} 1$.

[Win98] R. Winkel. On the multiplication of Schubert polynomials. Adv. in Appl. Math., 20(1):73-97, 1998. 
